\title{
Empathy and Social Attribution Skills Moderate the Relationship between Temporal Lobe Volume and Facial Expression Recognition Ability in Schizophrenia
}

\author{
Minjee Jung ${ }^{1}$, Seung Yeon Baik', Yourim Kim¹, Sungkean Kim', Dongil Min', Jeong-Youn Kim¹, \\ Seunghee Won ${ }^{2}$, Seung-Hwan Lee ${ }^{1,3}$ \\ ${ }^{1}$ Department of Psychiatry, Clinical Emotion and Cognition Research Laboratory, Inje University College of Medicine, Goyang, ${ }^{2}$ Department \\ of Psychiatry, Kyungpook National University Hospital, Daegu, ${ }^{3}$ Department of Psychiatry, Ilsan Paik Hospital, Inje University College of \\ Medicine, Goyang, Korea
}

\begin{abstract}
Objective: While impaired facial expression recognition has been closely associated with reduced temporal lobe volume in patients with schizophrenia, this study aimed at examining whether empathy and social attribution affect such a relationship.

Methods: A total of 43 patients with schizophrenia and 43 healthy controls underwent a facial expression recognition task (FERT) and magnetic resonance imaging. Basic empathy scale and the social attribution task-multiple choice were used to measure empathy and social attribution.

Results: Patients with schizophrenia showed significant positive correlations between the total temporal lobe volume and the FERT-accuracy (FERT-ACC). Diminished temporal lobe volume predicted the impaired facial emotion recognition ability. Both empathy and social attribution played roles as moderators of the path from the left amygdala volume, left fusiform gyrus volume, both sides of the superior temporal gyrus volume, and left middle temporal gyrus volume to the FERT-ACC. In contrast, empathy alone functioned as a moderator between the right fusiform gyrus volume, right middle temporal gyrus volume, and FERT-ACC. No significant interaction was found for healthy controls.

Conclusion: Our results suggest that social cognition remediation training on empathy and social attribution, could buffer the negative effects of small temporal lobe volume on interpersonal emotional communication in patients with schizophrenia.
\end{abstract}

KEY WORDS: Schizophrenia; Temporal lobe; Social cognitive ability; Empathy; Social attribution; Facial emotion recognition task.

\section{INTRODUCTION}

The ability to identify and interpret other people's facial expressions is crucial in our daily life. However, numerous studies have reported that patients with schizophrenia show impairment in the recognition of facial emotions, as well as in the judgement and labeling of facial expressions, recognition memory for unfamiliar faces, and

Received: July 5, 2019/ Revised: October 21, 2019

Accepted: November 5, 2019

Address for correspondence: Seung-Hwan Lee

Department of Psychiatry, Ilsan Paik Hospital, Inje University

College of Medicine, 170 Juhwa-ro, Ilsanseo-gu, Goyang 10380,

Korea

E-mail: Ishpss@paik.ac.kr

ORCID: https://orcid.org/0000-0003-0305-3709 identification of facial identity [1-7]. The difficulties in emotional recognition in schizophrenia have been explained by brain structural abnormalities [8-11]. In particular, temporal lobe dysfunction was reported to be a core element of the deficit in facial emotion recognition in patients with schizophrenia $[12,13]$. Specifically, the temporal lobe areas associated with facial emotion recognition include the hippocampus [14], amygdala [15-17], fusiform gyrus [18-21], superior temporal [22], and middle temporal [23] regions. Furthermore, the fusiform gyrus, hippocampus, temporal areas, and amygdala regions were all demonstrated to be structurally smaller in patients with schizophrenia than in healthy controls [8,24-30]. Such findings suggest that diminished temporal

(ㄷ) This is an Open-Access article distributed under the terms of the Creative Commons Attribution Non-Commercial License (http://creativecommons.org/licenses/by-nc/4.0) which permits unrestricted non-commercial use, distribution, and reproduction in any medium, provided the original work is properly cited. 
lobe volume plays a role in the impaired facial emotion recognition in patients with schizophrenia.

Facial emotion recognition deficits have been studied in the context of social cognition, or the mental mechanisms associated with perceiving, understanding, and interpreting the social world [31], in patients with schizophrenia [32]. Social cognition can be divided into three domains: 1) the ability to perceive social information, 2) understanding of other's cognitive or affective states and 3) decision-making while considering other's goals [33]. In this perspective, facial recognition ability falls into the first domain, social perception. While patients with schizophrenia show deficits in all three domains [6,34,35], previous studies have consistently demonstrated that the lack of facial emotion recognition is related to reduced temporal lobe volume. In particular, a review stated that abnormalities in face processing in schizophrenia are strongly associated with structural abnormalities in regions such as fusiform gyrus, superior temporal sulcus, amygdala and prefrontal cortex [36].

On the other hand, the current study also focused on the role of empathy and social attribution, which are incorporated in the second domain of social cognition, on the relationship between diminished temporal lobe volume and facial emotion recognition. Empathy is one of the most important personality traits that plays a role in social interaction and communication [37]. Also, social attribution, or mental state attribution, denotes the cognitive capacity to reflect upon one's own and others' mental states such as beliefs, desires, feelings and intention [38]. While several studies have shown that empathic or social attribution ability and social perception are independent constructs $[39,40]$, other studies proposed that the level of empathic ability could affect facial emotion processing [37]. In addition, given that some social cognition programs (e.g., a computerized program practicing recognition of others' mental states) have shown a positive effect on facial affect recognition [41], it is assumed that enhancing empathic or social attribution ability would attenuate the effect of reduced temporal lobe volume on facial emotion recognition. Based on these previous findings, we hypothesized that empathy or social attribution ability could moderate the relationship between diminished temporal lobe volume and abnormalities in facial emotion recognition in schizophrenia.

In this study, we attempted to investigate whether em- pathy and social attribution, constructs that involves social understanding domain of social cognitive ability, would buffer the relationship between temporal lobe volume and facial emotion perception skills in schizophrenia. Our hypothesis was that 1) a diminished temporal lobe volume would be associated with a deficit in facial emotion recognition in schizophrenia; and 2) empathy and social attribution, would act as moderators of the relationship between the temporal lobe volume and facial expression recognition in schizophrenia.

\section{METHODS}

\section{Participants}

A total of 86 subjects, who were recruited from both the Ilsan Paik hospital and local communities, participated in this study. The subject pool was comprised of patients with schizophrenia as well as healthy controls (Table 1). All patients were assessed for Axis I disorders by a psychiatrist, based on the Structured Clinical Interview for the Diagnostic and Statistical Manual of Mental Disorders, 4th edition. None of the patients had a lifetime history of central nervous system disease, alcohol or drug abuse, mental retardation, or head injury with loss of consciousness. All patients with schizophrenia were undergoing treatment with atypical antipsychotics. The exclusion criteria for the initial screening included identifiable neurological disorders, head injury, and any personal or family history of psychiatric illness. Healthy controls had no prior or present mental disorder. All participants provided a written informed consent prior to their enrollment in the investigation (Ispaik-2018-11-006).

\section{Measures}

\section{$M R I$ acquisition and volume}

A 1.5 T scanner was used to conduct magnetic resonance imaging (MRI) (Magneton Avanto; Siemens, Erlangen, Germany). Foam pads provided by the manufacturer were used to minimize head motion. High-resolution T1weighted MRI images were acquired with the following parameters: an acquisition matrix of $227 \times 384$, a field-ofview of $210 \times 250$, a voxel size of $0.9 \times 0.7 \times 1.2$, a total of 87,168 voxels, an echo time of $3.42 \mathrm{~ms}$, a repetition time of 1,900 ms, a 1.2-mm slice thickness, and a flip angle of $15^{\circ}$. 
Table 1. Participants' demographic information $(n=86)$

\begin{tabular}{|c|c|c|c|}
\hline Variable & Patients with schizophrenia $(n=43)$ & Healthy controls $(n=43)$ & $p$ value \\
\hline \multicolumn{4}{|l|}{ Demographics } \\
\hline \multicolumn{4}{|l|}{ Sex } \\
\hline Male & $16(37.2)$ & $20(46.5)$ & 0.382 \\
\hline Female & $27(62.8)$ & $23(53.5)$ & \\
\hline Age (yr) & $43.42 \pm 10.83$ & $39.93 \pm 13.21$ & 0.184 \\
\hline Education (yr) & $13.37 \pm 2.80$ & $14.58 \pm 3.67$ & 0.089 \\
\hline Pre-morbid IQ/IQ & $99.36 \pm 10.32$ & $107.97 \pm 8.58$ & $<0.001$ \\
\hline DOI (yr) & $12.87 \pm 8.34$ & - & \\
\hline CPZeq (mg) & $470.50 \pm 607.15$ & - & \\
\hline \multicolumn{4}{|l|}{ Brain volume $\left(\mathrm{mm}^{3}\right)$} \\
\hline TIV & $1,542.59 \pm 156.45$ & $1,564.45 \pm 151.74$ & 0.513 \\
\hline LHV & $2.99 \pm 0.42$ & $3.26 \pm 0.42$ & 0.004 \\
\hline RHV & $3.32 \pm 0.59$ & $3.62 \pm 0.40$ & 0.008 \\
\hline LAV & $0.88 \pm 0.11$ & $0.96 \pm 0.10$ & $<0.001$ \\
\hline RAV & $0.85 \pm 0.14$ & $0.94 \pm 0.10$ & 0.001 \\
\hline LFV & $7.86 \pm 1.11$ & $8.40 \pm 1.21$ & 0.036 \\
\hline RFV & $7.59 \pm 1.25$ & $8.26 \pm 1.16$ & 0.008 \\
\hline LSTV & $5.93 \pm 0.90$ & $6.38 \pm 0.95$ & 0.026 \\
\hline RSTV & $6.33 \pm 0.92$ & $6.83 \pm 1.01$ & 0.019 \\
\hline LMTV & $13.54 \pm 1.96$ & $14.90 \pm 2.06$ & 0.002 \\
\hline RMTV & $13.57 \pm 2.05$ & $15.04 \pm 2.00$ & 0.001 \\
\hline \multicolumn{4}{|c|}{ FERT and social cognitive scales } \\
\hline BES & $66.78 \pm 8.67$ & $74.95 \pm 7.99$ & $<0.001$ \\
\hline SAT-MC & $8.81 \pm 4.79$ & $14.50 \pm 3.69$ & $<0.001$ \\
\hline FERT-ACC (\%) & $0.52 \pm 0.18$ & $0.73 \pm 0.12$ & $<0.001$ \\
\hline FERT-RT (ms) & $2,123.04 \pm 351.80$ & $1,740.74 \pm 267.04$ & $<0.001$ \\
\hline
\end{tabular}

Values are presented as number $(\%)$ or mean \pm standard deviation.

DOI, duration of illness; CPZeq, chlorpromazine equivalent; TIV, total intracranial volume; LHV, left hippocampus volume; RHV, right hippocampus volume; LAV, left amygdala volume; RAV, right amygdala volume; LFV, left fusiform gyrus volume; RFV, right fusiform gyrus volume; LSTV, left superior temporal gyrus volume; RSTV, right superior temporal gyrus volume; LMTV, left middle temporal gyrus volume; RMTV, right middle temporal gyrus volume; BES, basic empathy scale; SAT-MC, social attribution task-multiple choice; FERT-ACC, facial expression recognition task-accuracy; FERT-RT, facial expression recognition task-response time.

The voxel-based morphometry (VBM) was performed using the Computational Anatomy Toolbox (CAT12; developed by Christian Gaser, University of Jena, http://dbm. neuro.uni-jena.de/cat) with the SPM12 software package (Wellcome Department of Cognitive Neurology, London, UK) $[42,43]$. The structural $\mathrm{T} 1$ images were registered to an ICBM East Asian template and normalized using the DARTEL algorithm $[44,45]$. Thereafter, while all images were segmented into gray matter, white matter, and cerebrospinal fluid [45], they were modulated to obtain volume differences in gray matter using the Jacobian transformed tissue probability maps. The volume of the temporal lobe regions (left hippocampus volume, LHV; right hippocampus volume, RHV; left amygdala volume, LAV; right amygdala volume, RAV; left fusiform gyrus volume, LFV; right fusiform gyrus volume, RFV; left superior temporal gyrus volume, LSTV; right superior temporal gyrus volume, RSTV; left middle temporal gyrus volume, LMTV; and right middle temporal gyrus volume, RMTV) was extracted using the Neuromorphometrics atlas, available in SPM 12 (http://neuromorphometrics.com) (Fig. 1). These regions of interest were determined based on the regions that were found to be associated with facial recognition and schizophrenia in previous studies [13-31].

\section{Basic empathy scale (BES)}

The BES, a 5-point 20-item scale developed by Jolliffe and Farrington [46], was used to measure the levels of empathy. This scale consists of two sub-constructs: cognitive empathy and affective empathy. The total score ranges from 20 to 100, higher scores indicating greater levels of empathy. In this study, the whole scale revealed an excellent internal consistency ( $\alpha=0.87$ ), while the cognitive empathy ( $\alpha=0.79)$ and the affective empathy subscales 


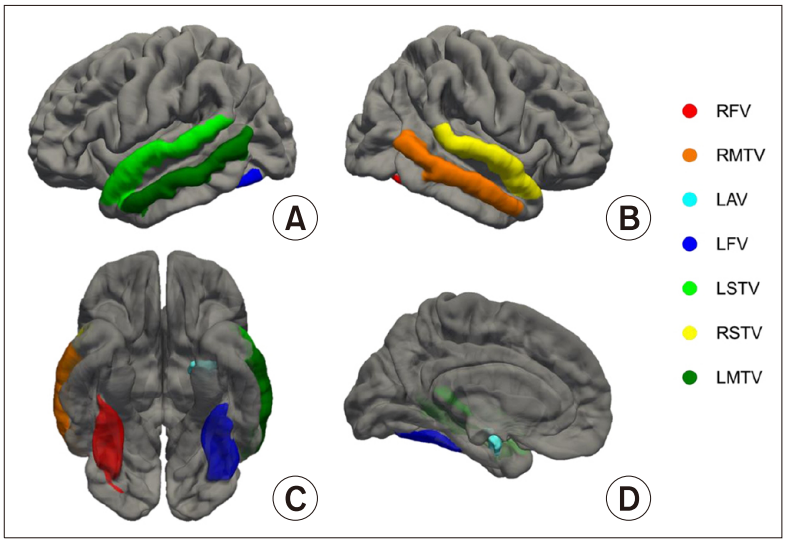

Fig. 1. Regions of interest for examination of the temporal lobe volume and FERT-ACC in patients with schizophrenia shown in (A) left view, (B) right view, (C) bottom view, and (D) left hemisphere. FERT-ACC, facial expression recognition task-accuracy; RFV, right fusiform gyrus volume; RMTV, right middle temporal gyrus volume; LAV, left amygdala volume; LFV, left fusiform gyrus volume; LSTV, left superior temporal gyrus volume; RSTV, right superior temporal gyrus volume; LMTV, left middle temporal gyrus volume.

( $\alpha=0.79$ ) demonstrated acceptable reliability.

\section{Social attribution task-multiple choice (SAT-MC)}

The social attribution test stimulus is based on the work conducted by Heider and Simmel [47] and later adapted by Klin $[48,49]$. The SAT-MC consists of a 64-second animation of a social drama enacted by a large triangle, small triangle, and a small circle (see original on www.youtube.com under "Heider and Simmel Movie"). After the participants watched the entire animation twice, they were presented 19 multiple-choice questions. Each question was presented with the relevant segment of the animation with 4 answer choices ( 1 choice depicting the correct emotional intent, 2 choices depicting incorrect emotional intent, and 1 choice on object motion without any emotional intent).

\section{Facial expression recognition task (FERT)}

This study used a facial expression recognition test that was modified from the facial affect labeling task [50]. It is a forced-choice emotional identification task that involves the presentation of the Korean Facial Expressions of Emotion [51] faces on the experimental laptop. While such faces were displayed on the left side of the computer screen, the response buttons (rectangles of $5.5 \times 3.5 \mathrm{~cm}$ ) appeared on the right side of the screen following the disappearance of the face. The distance between the re- sponse buttons was $0.5 \mathrm{~cm}$, and they were aligned equidistantly in a circular formation around the 4-cm central circle. The order of the response choices was fixed and was as follows: from the top right moving clockwise; happy, neutral, sad, surprised, fearful, disgusted, contempt, and angry. Participants were informed of the presence of such eight specific emotions during the task and were asked to provide their response by pressing the button on the screen that corresponded to the emotion displayed with a mouse. After pressing the response button, participants were instructed to quickly place the mouse back on the central circle and to wait. The picture was shown randomly within a block (i.e., a total of sixteen facial emotions). Each subject performed 2 practice blocks and 4 blocks in the original task. The original task consisted of four blocks, separated by an un-timed rest period to prevent fatigue. Participants completed the task in approximately 10 minutes. While the face appeared within 750 $\mathrm{ms}$, the inter-trial interval started at 3,000 $\mathrm{ms}$ from the reaction time (RT) and was added to the 1,500 ms of feedback. Immediately after the presentation of each face, a white screen was displayed. Subjects were asked to answer as fast as possible and both the answer accuracy and RT for correct responses were assessed for each emotion. Furthermore, the hit rate (number of correct trials/total number of trials) and RT (time taken to respond with the correct answer) were obtained.

\section{Data Analyses}

The Pearson's correlation analysis with bootstrapping at a 5,000-sampling rate was performed to examine the correlations among the variables. Moderating analysis was conducted using the SPSS Macro PROCESS for SPSS 2.16.3 (model number 1) developed by Preacher and Hayes [52]. Finally, the indirect effects were estimated for the variables of interest using both a normal theory approach and non-parametric bootstrapping to provide confidence intervals. Furthermore, the Johnson - Neyman technique was applied to investigate the regions in which the moderating variable had significant effects. Confidence intervals that contain the zero value indicate a non-significant conditional indirect effect and therefore imply a significant moderating effect. In contrast, confidence intervals that do not include the zero value describe a significant conditional indirect effect and a non-significant moderating effect. All significance levels were set at $p<0.05$ (two-tailed). 


\section{RESULTS}

\section{Descriptive Statistics}

The demographic information as well as the psychological characteristics of patients with schizophrenia and healthy controls is presented in Table 1.

For the correlations between the main variables, significant positive correlations were identified between all the temporal lobe areas and the FERT-ACC (LHV: $r=0.42$, $p<0.01$; RHV: $r=0.37, p<0.05$; LAV: $r=0.52, p<$ 0.01 ; RAV: $r=0.41, p<0.01$; LFV: $r=0.42, p<0.01$; RFV: $r=0.38, p<0.05$; LSTV: $r=0.39, p<0.05$; RSTV: $r=0.40, p<0.01$; LMTV: $r=0.44, p<0.01$; RMTV: $r=$ $0.42, p<0.01$ ), between the FERT-ACC and the BES score $(r=0.46, p<0.01)$, as well as between the FERT-ACC and the SAT-MC score $(r=0.53, p<0.001)$. In addition, the FERT-RT was negatively correlated with LMTV, RMTV, and FERT-ACC ( $r=-0.32, p<0.05 ; r=$ $-0.34, p<0.05 ; r=-0.59, p<0.001$, respectively). However, there was no significant relationship between all temporal lobe volume and BES and SAT-MC score except LAV $(r=0.31, p<0.05 ; r=0.47, p<0.01$, respectively).

With regard to the healthy controls, a significant positive correlation between the FERT-ACC and BES score $(r=$ $0.38, p<0.05)$, as well as between the FERT-ACC and SAT-MC score $(r=0.32, p<0.05)$ was observed. Finally, the FERT-RT was negatively associated with LAV, LFV, RFV, RSTV, and FERT-ACC $(r=-0.32, p<0.05 ; r=-0.31$, $p<0.05 ; r=-0.41, p<0.01 ; r=-0.35, p<0.05 ; r=$ $-0.57, p<0.001$, respectively). However, in line with patients with schizophrenia, significant relationship was not found between all temporal lobe volume and BES and SAT-MC score.

\section{Moderation Effects}

To examine the interaction between the temporal lobe volume and the social cognitive abilities in the FERT, moderation analyses were performed. Sex, age, years of education, pre-morbid IQ, illness duration, chlorpromazine equivalent and TIV were set as covariates. Our results indicated the direction of the interaction between the temporal lobe volume and the social cognitive abilities to be consistent with our hypothesis (Fig. 2).

\section{Interaction between temporal lobe volume and BES}

After controlling for the covariates, the LAV positively predicted the FERT-ACC $(\mathrm{B}=3.83, t=2.85, p=0.008)$, while the BES also positively predicted the FERT-ACC $(B=$ $0.05, t=2.48, p=0.019)$ in patients with schizophrenia. These results identified a significant direct effect of both the LAV and BES on the FERT-ACC. Furthermore, the interaction between $\mathrm{LAV}$ and $\mathrm{BES}$ had a significant effect on the FERT-ACC ( $\mathrm{B}=-0.05, t=-2.42, p=0.022)$, and the $R^{2}$ was increased due to the interaction $\left(\Delta R^{2}=0.06, \Delta F=\right.$ 5.84, $p=0.022$ ). Specifically, those participants who demonstrated high levels of BES performed better in the FERT-ACC, regardless of their diminished LAV.

Similarly, after controlling for covariates, the LSTV positively predicted the FERT-ACC ( $\mathrm{B}=0.66, t=2.79, p=$ 0.009 ), while the BES positively predicted the FERT-ACC ( $\mathrm{B}=0.06, t=2.80, p=0.009)$ in patients with schizophrenia. These results indicated a significant direct effect of both the LSTV and BES on the FERT-ACC. Furthermore, the interaction between LSTV and BES had a significant effect on the FERT-ACC ( $\mathrm{B}=-0.01, t=-2.67, p=0.012)$, and the $R^{2}$ was increased due to the interaction $\left(\Delta R^{2}=0.07, \Delta F=\right.$ $7.12, p=0.012$ ).

Similar significant interactions were found in other temporal lobe areas, including the LFV, RFV, RSTV, LMTV,
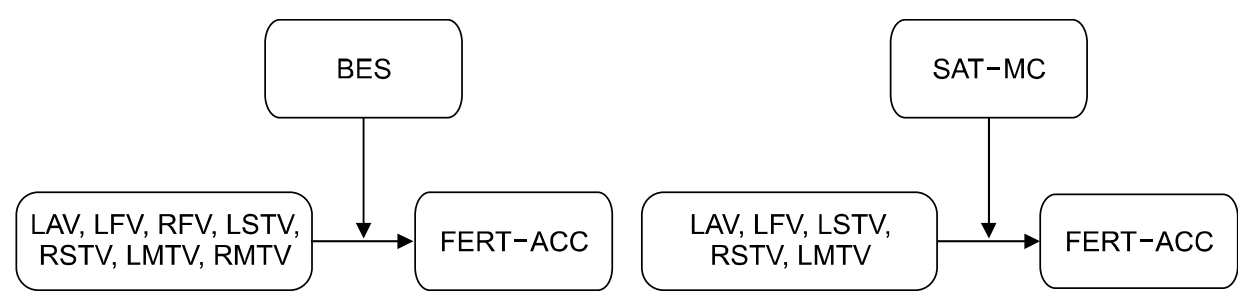

Fig. 2. BES and SAT-MC as moderators of the relationship between the temporal lobe volume and FERT-ACC in patients with schizophrenia. LAV, left amygdala volume; LFV, left fusiform gyrus volume; RFV, right fusiform gyrus volume; LSTV, left superior temporal gyrus volume; RSTV, right superior temporal gyrus volume; LMTV, left middle temporal gyrus volume; RMTV, right middle temporal gyrus volume; BES, basic empathy scale; SAT-MC, social attribution task-multiple choice; FERT-ACC, facial expression recognition task-accuracy. 

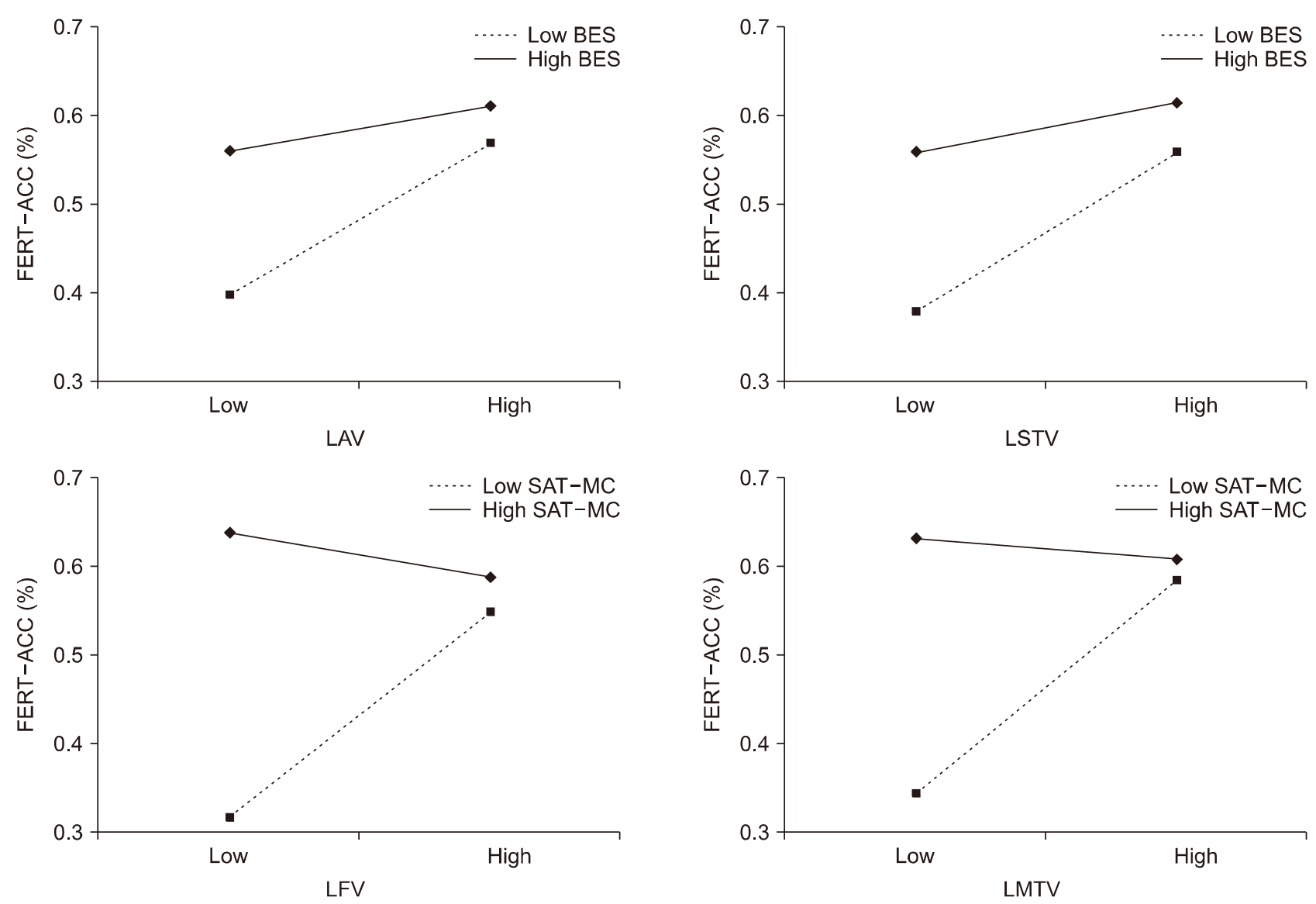

Fig. 3. Interaction effects between the temporal lobe volume and social cognitive abilities on prediction of the FERT-ACC in patients with schizophrenia.

LAV, left amygdala volume; LFV, left fusiform gyrus volume; LSTV, left superior temporal gyrus volume; LMTV, left middle temporal gyrus volume; FERT-ACC, facial expression recognition task-accuracy; BES, basic empathy scale; SAT-MC, social attribution task-multiple choice.

and RMTV. In contrast, an absence of significant results was found between the LHV, RHV, RAV and the FERT-ACC in patients with schizophrenia. See Table 2 for the complete results on the interaction between temporal lobe volume and BES. The examples of interactions were plotted in Figure 3.

\section{Interaction between temporal lobe volume and SAT-MC}

After controlling for the covariates, the LFV positively predicted the FERT-ACC ( $\mathrm{B}=0.09, t=2.70, p=0.011$ ), while also the SAT-MC positively predicted the FERTACC (B $=0.08, t=2.83, p=0.008)$ in patients with schizophrenia. These results suggest a significant direct effect of both the LFV and SAT-MC on the FERT-ACC. In addition, the interaction between the LFV and SAT-MC had a significant effect on the FERT-ACC ( $B=-0.01, t=$
$-2.23, p=0.033)$, while the $\mathrm{R}^{2}$ was increased due to the interaction $\left(\Delta \mathrm{R}^{2}=0.03, \Delta \mathrm{F}=4.97, p=0.033\right)$. Therefore, those participants who demonstrated high levels of SAT-MC performed better in the FERT-ACC, regardless of their diminished LFV.

Similarly, after controlling for the covariates, the LMTV positively predicted the FERT-ACC $(\mathrm{B}=0.06, t=2.83, p=$ $0.008)$, while the SAT-MC also positively predicted the FERT-ACC ( $\mathrm{B}=0.09, t=3.32, p=0.002)$ in patients with schizophrenia. These results indicated a significant direct effect of both the LMTV and SAT-MC on the FERT-ACC. Furthermore, the interaction between the LMTV and SAT$M C$ had a significant effect on the FERT-ACC $(B=-0.01$, $t=-2.71, p=0.011)$, while the $\mathrm{R}^{2}$ was increased due to the interaction $\left(\Delta \mathrm{R}^{2}=0.05, \Delta \mathrm{F}=7.32, p=0.011\right)$. See Figure 2 for interaction graphs of the selected results.

Significant interactions with the SAT-MC were also 
Table 2. Regression analysis examining moderation effects between temporal lobe volume and social cognitive abilities on FERT-ACC in schizophrenia $(n=43)$

\begin{tabular}{|c|c|c|c|c|c|c|c|c|c|c|}
\hline \multirow{2}{*}{ Predictor } & \multicolumn{5}{|c|}{ Left } & \multicolumn{5}{|c|}{ Right } \\
\hline & B & SE & $t$ & $p$ value & $\mathrm{R}^{2}$ & B & SE & $t$ & $p$ value & $R^{2}$ \\
\hline \multicolumn{11}{|l|}{ Amygdala volume } \\
\hline Constant & -3.65 & 1.27 & -2.88 & 0.007 & 0.70 & -1.91 & 1.29 & -1.48 & 0.148 & 0.61 \\
\hline Sex & 0.11 & 0.05 & 2.26 & 0.031 & & 0.068 & 0.05 & 1.29 & 0.207 & \\
\hline Age & -0.00 & 0.00 & -1.91 & 0.065 & & -0.01 & 0.00 & -3.21 & 0.003 & \\
\hline Education & 0.01 & 0.01 & 0.76 & 0.456 & & 0.01 & 0.00 & 0.87 & 0.392 & \\
\hline Pre-morbid IQ & 0.01 & 0.00 & 2.83 & 0.008 & & 0.01 & 0.00 & 2.58 & 0.015 & \\
\hline DOI & -0.00 & 0.00 & -0.78 & 0.441 & & 0.00 & 0.00 & 0.07 & 0.944 & \\
\hline CPZeq & -0.00 & 0.00 & -1.81 & 0.080 & & -0.00 & 0.00 & -1.83 & 0.076 & \\
\hline TIV & 0.00 & 0.00 & 0.35 & 0.729 & & 0.00 & 0.00 & 0.59 & 0.556 & \\
\hline LAV & 3.83 & 1.34 & 2.85 & 0.008 & & & & & & \\
\hline RAV & & & & & & 1.76 & 1.36 & 1.30 & 0.205 & \\
\hline BES & 0.05 & 0.02 & 2.48 & 0.019 & & 0.03 & 0.02 & 1.34 & 0.189 & \\
\hline $\mathrm{LAV} * \mathrm{BES}$ & -0.05 & 0.02 & -2.42 & 0.022 & 0.76 & & & & & \\
\hline $\mathrm{RAV} * \mathrm{BES}$ & & & & & & -0.03 & 0.00 & -2.27 & 0.030 & 0.63 \\
\hline \multicolumn{11}{|c|}{ Fusiform gyrus volume } \\
\hline Constant & -3.27 & 1.40 & -2.33 & 0.026 & 0.77 & -3.14 & 1.24 & -2.52 & 0.017 & 0.67 \\
\hline Sex & 0.059 & 0.05 & 1.18 & 0.245 & & 0.06 & 0.05 & 1.15 & 0.258 & \\
\hline Age & -0.01 & 0.00 & -2.87 & 0.007 & & -0.01 & 0.00 & -3.57 & 0.001 & \\
\hline Education & 0.01 & 0.01 & 1.51 & 0.140 & & 0.01 & 0.01 & 1.40 & 0.173 & \\
\hline Pre-morbid IQ & 0.01 & 0.00 & 2.28 & 0.029 & & 0.00 & 0.00 & 2.84 & 0.008 & \\
\hline DOI & 0.00 & 0.00 & 0.16 & 0.875 & & 0.00 & 0.00 & 0.19 & 0.852 & \\
\hline CPZeq & -0.00 & 0.00 & -2.13 & 0.041 & & -0.00 & 0.00 & -2.02 & 0.052 & \\
\hline TIV & 0.00 & 0.00 & 0.29 & 0.773 & & 0.00 & 0.00 & 0.74 & 0.464 & \\
\hline LFV & 0.37 & 0.17 & 2.19 & 0.036 & & & & & & \\
\hline RFV & & & & & & 0.33 & 0.15 & 2.25 & 0.031 & \\
\hline BES & 0.05 & 0.02 & 2.23 & 0.033 & & 0.05 & 0.02 & 2.44 & 0.021 & \\
\hline LFV*BES & -0.01 & 0.00 & -2.08 & 0.045 & 0.81 & & & & & \\
\hline RFV*BES & & & & & & -0.01 & 0.00 & -2.27 & 0.030 & 0.74 \\
\hline \multicolumn{11}{|c|}{ Superior temporal gyrus volume } \\
\hline Constant & -4.25 & 1.46 & -2.91 & 0.007 & 0.67 & -3.94 & 1.55 & -2.54 & 0.016 & 0.66 \\
\hline Sex & 0.08 & 0.05 & 1.71 & 0.100 & & 0.082 & 0.05 & 1.67 & 0.104 & \\
\hline Age & -0.01 & 0.00 & -2.69 & 0.011 & & -0.01 & 0.00 & -2.72 & 0.011 & \\
\hline Education & 0.01 & 0.01 & 1.65 & 0.110 & & 0.01 & 0.01 & 1.57 & 0.126 & \\
\hline Pre-morbid IQ & 0.01 & 0.00 & 2.45 & 0.020 & & 0.01 & 0.00 & 2.46 & 0.020 & \\
\hline DOI & -0.00 & 0.00 & -0.21 & 0.835 & & -0.00 & 0.00 & -0.40 & 0.694 & \\
\hline CPZeq & 0.00 & 0.00 & -1.52 & 0.138 & & 0.00 & 0.00 & -1.50 & 0.143 & \\
\hline TIV & 0.00 & 0.00 & -0.11 & 0.913 & & 0.00 & 0.00 & -0.23 & 0.819 & \\
\hline LSTV & 0.66 & 0.24 & 2.79 & 0.009 & & & & & & \\
\hline RSTV & & & & & & 0.58 & 0.23 & 2.50 & 0.018 & \\
\hline BES & 0.06 & 0.02 & 2.80 & 0.009 & & 0.05 & 0.02 & 2.42 & 0.021 & \\
\hline LSTV*BES & -0.01 & 0.00 & -2.67 & 0.012 & 0.74 & & & & & \\
\hline RSTV*BES & & & & & & -0.01 & 0.00 & -2.32 & 0.027 & 0.72 \\
\hline \multicolumn{11}{|c|}{ Middle temporal gyrus volume } \\
\hline Constant & -2.92 & 1.27 & -2.30 & 0.028 & 0.64 & -3.50 & 1.28 & -2.74 & 0.010 & 0.65 \\
\hline Sex & 0.07 & 0.05 & 1.46 & 0.153 & & 0.08 & 0.05 & 1.53 & 0.136 & \\
\hline Age & -0.01 & 0.00 & -2.52 & 0.017 & & -0.01 & 0.00 & -2.74 & 0.010 & \\
\hline Education & 0.01 & 0.01 & 1.39 & 0.173 & & 0.01 & 0.01 & 1.27 & 0.215 & \\
\hline Pre-morbid IQ & 0.01 & 0.00 & 2.56 & 0.015 & & 0.01 & 0.00 & 2.76 & 0.010 & \\
\hline DOI & -0.00 & 0.00 & -0.15 & 0.883 & & -0.00 & 0.00 & -0.02 & 0.983 & \\
\hline CPZeq & 0.00 & 0.00 & -1.33 & 0.194 & & -0.00 & 0.00 & -2.03 & 0.051 & \\
\hline TIV & 0.00 & 0.00 & -0.00 & 0.999 & & 0.00 & 0.00 & 0.80 & 0.431 & \\
\hline LMTV & 0.19 & 0.09 & 2.17 & 0.038 & & & & & & \\
\hline RMTV & & & & & & 0.21 & 0.09 & 2.45 & 0.020 & \\
\hline BES & 0.04 & 0.02 & 2.17 & 0.038 & & 0.05 & 0.02 & 2.66 & 0.012 & \\
\hline LMTV*BES & -0.00 & 0.00 & -2.02 & 0.052 & 0.69 & & & & & \\
\hline RMTV*BES & & & & & & -0.00 & 0.00 & -2.49 & 0.018 & 0.72 \\
\hline
\end{tabular}

FERT-ACC, facial expression recognition task-accuracy; SE, standard error; DOI, durationof illness CPZeq, chlorpromazine equivalent; TIV, total intracranial gyrus volume; LAV, left amygdala volume; RAV, right amygdala volume; BES, basic empathy scale; LFV, left fusiform gyrus volume; RFV, right fusiform gyrus volume; LSTV, left superior temporal gyrus volume; RSTV, right superior temporal gyrus volume; LMTV, left middle temporal gyrus volume; RMTV, right middle temporal gyrus volume. 
Table 3. Regression analysis examining moderation effects between temporal lobe volume and SAT-MC on FERT-ACC in schizophrenia $(n=43)$

\begin{tabular}{|c|c|c|c|c|c|c|c|c|c|c|}
\hline \multirow{2}{*}{ Predictor } & \multicolumn{5}{|c|}{ Left } & \multicolumn{5}{|c|}{ Right } \\
\hline & B & SE & $t$ & $p$ value & $\mathrm{R}^{2}$ & B & SE & $t$ & $p$ value & $R^{2}$ \\
\hline \multicolumn{11}{|l|}{ Amygdala volume } \\
\hline Constant & -1.05 & 0.36 & -2.90 & 0.007 & 0.70 & -0.27 & 0.31 & -0.85 & 0.402 & 0.73 \\
\hline Sex & 0.09 & 0.04 & 2.42 & 0.022 & & 0.07 & 0.04 & 1.70 & 0.097 & \\
\hline Age & -0.01 & 0.00 & -3.31 & 0.002 & & -0.01 & 0.00 & -4.23 & 0.000 & \\
\hline Education & 0.00 & 0.01 & 0.54 & 0.592 & & 0.01 & 0.01 & 1.46 & 0.15 & \\
\hline Pre-morbid IQ & 0.01 & 0.00 & 3.09 & 0.004 & & 0.01 & 0.00 & 2.61 & 0.014 & \\
\hline DOI & -0.00 & 0.00 & -1.26 & 0.215 & & -0.00 & 0.00 & -0.99 & 0.325 & \\
\hline CPZeq & -0.00 & 0.00 & -2.73 & 0.010 & & -0.00 & 0.00 & -2.95 & 0.006 & \\
\hline TIV & 0.00 & 0.00 & 0.32 & 0.748 & & 0.00 & 0.00 & 0.54 & 0.593 & \\
\hline LAV & 1.10 & 0.33 & 3.39 & 0.002 & & & & & & \\
\hline RAV & & & & & & 0.13 & 0.19 & 0.69 & 0.497 & \\
\hline SAT-MC & 0.09 & 0.03 & 3.62 & 0.001 & & 0.04 & 0.02 & 1.71 & 0.097 & \\
\hline LAV*SAT-MC & -0.09 & 0.03 & -3.09 & 0.004 & 0.76 & & & & & \\
\hline RAV*SAT-MC & & & & & & -0.03 & 0.29 & -1.07 & 0.294 & 0.74 \\
\hline \multicolumn{11}{|c|}{ Fusiform gyrus volume } \\
\hline Constant & -0.56 & 0.32 & -1.74 & 0.091 & 0.77 & -0.33 & 0.31 & -1.05 & 0.300 & 0.86 \\
\hline Sex & 0.06 & 0.04 & 1.40 & 0.171 & & 0.07 & 0.04 & 1.67 & 0.105 & \\
\hline Age & -0.01 & 0.00 & -3.52 & 0.001 & & -0.01 & 0.00 & -4.17 & 0.000 & \\
\hline Education & 0.01 & 0.01 & 1.32 & 0.196 & & 0.01 & 0.01 & 1.39 & 0.176 & \\
\hline Pre-morbid IQ & 0.00 & 0.00 & 2.480 & 0.018 & & 0.01 & 0.00 & 2.62 & 0.014 & \\
\hline DOI & -0.00 & 0.00 & -1.15 & 0.260 & & -0.00 & 0.00 & -0.81 & 0.423 & \\
\hline CPZeq & -0.00 & 0.00 & -2.66 & 0.012 & & -0.00 & 0.00 & -2.96 & 0.006 & \\
\hline TIV & -0.00 & 0.00 & -0.70 & 0.489 & & 0.00 & 0.00 & -0.40 & 0.697 & \\
\hline LFV & 0.09 & 0.04 & 2.70 & 0.011 & & & & & & \\
\hline RFV & & & & & & 0.03 & 0.03 & 1.09 & 0.283 & \\
\hline SAT-MC & 0.08 & 0.03 & 2.83 & 0.008 & & 0.05 & 0.02 & 2.29 & 0.029 & \\
\hline LFV*SAT-MC & -0.01 & 0.00 & -2.23 & 0.033 & 0.81 & & & & & \\
\hline RFV*SAT-MC & & & & & & -0.00 & 0.00 & -1.59 & 0.121 & 0.88 \\
\hline \multicolumn{11}{|c|}{ Superior temporal gyrus volume } \\
\hline Constant & -0.55 & 0.31 & -1.75 & 0.089 & 0.77 & -0.70 & 0.32 & -2.19 & 0.036 & 0.79 \\
\hline Sex & 0.06 & 0.04 & 1.51 & 0.140 & & 0.06 & 0.04 & 1.52 & 0.139 & \\
\hline Age & -0.01 & 0.00 & -3.41 & 0.002 & & -0.01 & 0.00 & -3.94 & 0.000 & \\
\hline Education & 0.01 & 0.01 & 1.31 & 0.200 & & 0.00 & 0.01 & 0.42 & 0.680 & \\
\hline Pre-morbid IQ & 0.00 & 0.00 & 2.40 & 0.022 & & 0.00 & 0.00 & 3.41 & 0.001 & \\
\hline DOI & -0.00 & 0.00 & -0.62 & 0.541 & & -0.00 & 0.00 & -1.24 & 0.224 & \\
\hline CPZeq & -0.00 & 0.00 & -2.57 & 0.015 & & -0.00 & 0.00 & -2.38 & 0.024 & \\
\hline TIV & -0.00 & 0.00 & -0.60 & 0.550 & & -0.00 & 0.00 & -0.90 & 0.377 & \\
\hline LSTV & 0.12 & 0.05 & 2.43 & 0.021 & & & & & & \\
\hline RSTV & & & & & & 0.14 & 0.05 & 3.16 & 0.004 & \\
\hline SAT-MC & 0.07 & 0.02 & 3.07 & 0.004 & & 0.09 & 0.03 & 3.36 & 0.002 & \\
\hline LSTV*SAT-MC & -0.01 & 0.00 & -2.41 & 0.022 & 0.81 & & & & & \\
\hline RSTV*SAT-MC & & & & & & -0.01 & 0.00 & -2.76 & 0.010 & 0.84 \\
\hline \multicolumn{11}{|c|}{ Middle temporal gyrus volume } \\
\hline Constant & -0.68 & 0.32 & -2.12 & 0.042 & 0.78 & -0.38 & 0.33 & -1.18 & 0.247 & 0.86 \\
\hline Sex & 0.06 & 0.04 & 1.56 & 0.128 & & 0.07 & 0.44 & 1.59 & 0.122 & \\
\hline Age & -0.01 & 0.00 & -3.42 & 0.002 & & -0.01 & 0.00 & -3.37 & 0.002 & \\
\hline Education & 0.01 & 0.01 & 0.98 & 0.334 & & 0.01 & 0.01 & 1.11 & 0.277 & \\
\hline Pre-morbid IQ & 0.01 & 0.00 & 2.94 & 0.006 & & 0.01 & 0.00 & 2.65 & 0.012 & \\
\hline DOI & -0.00 & 0.00 & -1.15 & 0.260 & & -0.00 & 0.00 & -1.11 & 0.275 & \\
\hline CPZeq & -0.00 & 0.00 & -2.40 & 0.002 & & -0.00 & 0.00 & -2.66 & 0.012 & \\
\hline TIV & -0.00 & 0.00 & -0.40 & 0.689 & & 0.00 & 0.00 & 0.17 & 0.865 & \\
\hline LMTV & 0.06 & 0.02 & 2.83 & 0.008 & & & & & & \\
\hline RMTV & & & & & & 0.02 & 0.02 & 1.21 & 0.234 & \\
\hline SAT-MC & 0.09 & 0.03 & 3.32 & 0.002 & & 0.05 & 0.03 & 2.18 & 0.036 & \\
\hline LMTV*SAT-MC & -0.01 & 0.00 & -2.71 & 0.011 & 0.83 & & & & & \\
\hline RMTV*SAT-MC & & & & & & -0.00 & 0.00 & -1.53 & 0.135 & 0.88 \\
\hline
\end{tabular}

SAT-MC, social attribution task-multiple choice; FERT-ACC, facial expression recognition task-accuracy; SE, standard error; DOI, duration of illness; CPZeq, chlorpromazine equivalent; TIV, total intracranial volume; LAV, left amygdala volume; RAV, right amygdala volume; LFV, left fusiform gyrus volume; RFV, right fusiform gyrus volume; LSTV, left superior temporal gyrus volume; RSTV, right superior temporal gyrus volume; LMTV, left middle temporal gyrus volume; RMTV, right middle temporal gyrus volume. 
found at regions LAV, LSTV, and RSTV. In contrast, an absence of significant moderating effects was observed in the SAT-MC between the LHV, RHV, RAV, RFV, RMTV and the FERT-ACC in patients with schizophrenia. See Table 3 for the complete results on the interaction between temporal lobe volume and SAT-MC. The examples of interactions were plotted in Figure 3.

Moreover, moderating effects of either the BES or SAT-MC in the relationship between the temporal lobe volume and the FERT-ACC were not found in healthy controls. Finally, significant results were only seen in the FERT-ACC (in patients with schizophrenia), whereas an absence of significant moderation results was observed when using the FERT-RT as a dependent variable in both patients with schizophrenia and healthy controls.

\section{Probing an Interaction (Johnson-Neyman Analysis)}

The Johnson - Neyman analysis was performed to examine the regions in which the moderating variable had significant effects. Several significant results of the Johnson-Neyman analysis are reported below.

For the interaction effect between the LAV and BES on the FERT-ACC, the conditional indirect effect was significant when the raw score of the BES was below 66.31. Conversely, the conditional indirect effect was not significant when the raw score of the BES exceeded 66.31. Similarly, for the interaction effect between the LSTV and BES on the FERT-ACC, the conditional indirect effect was significant only when the raw score of the BES was below 62.53. This implies that in the case of a high BES, the levels of the FERT-ACC remain constant and do not change with the levels of the LAV and LSTV. Overall, the moderating effect was significant only in patients with schizophrenia with high BES (above 66.31 for LAV and 62.52 for LSTV).

For the interaction between LFV and SAT-MC on the FERT-ACC, the conditional indirect effect was significant when the raw score of the SAT-MC was below 6.96. Conversely, the conditional indirect effect was not significant when the raw score of the SAT-MC exceeded 6.96. For the interaction between LMTV and SAT-MC, the conditional indirect effect was significant when the raw score of the SAT-MC was below 5.21. This implies that in the case of a high SAT-MC, the levels of the FERT-ACC remain constant and do not change with the levels of the LFV and LMTV. Overall, the moderating effect was sig- nificant only in patients with schizophrenia with high SAT-MC (above 5.21 for LFV and 6.96 for LMTV).

\section{DISCUSSION}

In the current study, the relationship between the temporal lobe, social cognitive abilities, and facial expression recognition ability in patients with schizophrenia was investigated. Following are reported the main significant results: 1) Patients with schizophrenia showed a significantly reduced temporal lobe volume and poor performances in the FERT compared to healthy controls; 2) A significant positive correlation between the temporal lobe volume and the FERT-ACC was found only in patients with schizophrenia; 3) Diminished LAV, LFV, RFV, LSTV, RSTV, LMTV, RMTV predicted an impaired facial emotion recognition in patients with schizophrenia; 4) Both empathy and social attribution acted as moderators in the relationship between the LAV, LFV, LSTV, RSTV, and the FERT-ACC in patients with schizophrenia. Empathy alone functioned as a moderator on the relationship of the RFV and RMTV with the FERT-ACC in patients with schizophrenia. In contrast, an absence of moderation effects was observed in healthy controls.

The current study demonstrated that patients with schizophrenia have a diminished temporal lobe volume and an impaired facial affect recognition ability as opposed to healthy controls. This is in concordance with previous studies reporting a diminished temporal lobe volume in schizophrenia $[8,24,27-30,53]$. Furthermore, patients with schizophrenia were demonstrated to have severe deficits in their ability to make social judgements from facial expressions [6,54-56] and a dysfunctional neurophysiological processing for facial and affective facial stimuli $[57,58]$. Similarly, our study reported that patients with schizophrenia show a lower accuracy and slower speeds in the FERT compared to $\mathrm{HC}$, indicating a difficulty in the emotional processing.

In the current study, the temporal lobe volume showed a significant positive correlation with the recognition ability of the facial emotional expression in patients with schizophrenia. Given that the temporal lobe areas represent the main areas associated with the interpretation of face emotion [12], the diminished temporal lobe volume may lead to difficulties in judging face emotion in patients with schizophrenia. For example, a smaller amygdala has 
been regarded as a crucial factor explaining the poorer recognition of emotional expression $[5,59,60]$ and the impaired facial emotional learning [61] in patients with schizophrenia. Similarly, reduced fusiform and middle temporal gray matter volumes were also associated with facial emotion recognition deficits in patients with schizophrenia [12]. However, there was no significant correlation between temporal lobe volume and facial affect recognition in healthy control. This null result might be attributed to the fact that brain volume is not a major factor influencing information processing in healthy controls $[62,63]$.

In addition to the significant correlations between the temporal lobe volume and the FERT-ACC, the diminished LAV, LFV, RFV, LSTV, RSTV, LMTV, RMTV also predicted the impaired facial emotion recognition in patients with schizophrenia. These results are consistent with previous studies reporting an association between the temporal lobe volume and the facial emotion recognition ability in healthy subjects $[15,19,23]$, as well as in patients with schizophrenia [12]. With regard to the amygdala, only the LAV, and not the RAV, significantly predicted the impaired recognition of facial emotional expression. This result is in line with a previous study suggesting the left amygdala to be increasingly associated with emotional processing compared to the right amygdala [64-68]. For example, imaging studies on the reactions to positive and negative non-verbal emotional stimuli identified a predominantly left lateralization of the amygdala response $[65,66]$. In addition, meta-analyses suggested a greater left, as opposed to the right, amygdala activation during the cognitive processing of emotional stimuli $[64,68]$. Finally, an absence of significant relationships between both the left and right $\mathrm{HV}$ and facial expression recognition in patients with schizophrenia was observed. This may result from the fact that the hippocampus is more closely related to recall of memories, rather than their recognition. In fact, previous studies described that the hippocampus is required for the formation of episodic memories and that it plays an essential role in recollection-based memory (recall), rather than in familiarity-based memory (recognition) [69-71].

Further, empathy and social attribution were found to moderate the influence of the reduced temporal lobe volume on the FERT-ACC in patients with schizophrenia. Our results suggest that, despite the reduced volume of the temporal lobe, patients with schizophrenia with a higher level of empathy and social attribution show a higher recognition ability of facial expression. Furthermore, the role of social cognitive ability in the relationship between the brain structure and facial emotion recognition was revealed. Specifically, our findings are consistent with the existing literature indicating that social cognition remediation training could affect the facial emotion recognition as well as the changes in brain activity $[72,73]$.

In this respect, educating patients with schizophrenia with a biological deficit (e.g., reduced temporal lobe volume) on empathy and social attribution may be helpful for the improvement of face emotion recognition. In fact, several studies showed that training programs focusing on social cognition are effective in improving face emotion recognition in schizophrenia [74-79]. The results from these studies provide evidence of the beneficial effects of social cognition remediation training in schizophrenia by examining the underlying moderation mechanism. In this paper, we propose that training empathy and social attribution would be a more effective way for enhancing the ability to recognize facial emotion in patients with schizophrenia with abnormalities in their brain structures.

There are a few limitations of the present study. First, all the emotions were combined in this investigation and averaged in the FERT calculation. According to Kohler et al. [2], patients with schizophrenia performed worse in the recognition of fearful, disgusted, and neutral expressions than in the recognition of happy, sad, and angry expressions. Therefore, analyzing emotions separately in future studies may be meaningful. Second, the current study only theoretically examined the role of social cognitive abilities in the relationship between diminished temporal lobe volume and facial emotion recognition. The clinical implications would be greater if the real effect of social cognition remediation training on patients with schizophrenia with abnormalities in brain structure was investigated. Another limitation of this study is the small number of participants. Yet, it is compatible with group sizes from other studies of brain structure and facial emotion processing in patients $[5,12,80]$. Finally, this study used a cross-sectional design, and therefore only ensures a significant association among variables. We hope that the current research would pave the way for future research. 
The present findings suggest that empathy and social attribution are essential abilities that buffer against the effects of a diminished temporal lobe volume on the impaired facial expression recognition in patients with schizophrenia. Social cognition remediation training programs that enhance empathy and social attribution is suggested as an effective method for the improvement of social adaptation of patients with schizophrenia with smaller temporal brain volumes.

\section{Acknowledgments}

This work was supported by a grant from the Korea Science and Engineering Foundation (KOSEF), funded by the Korean government (NRF-2018R1A2A2A05018505).

\section{Conflicts of Interest}

No potential conflict of interest relevant to this article was reported.

\section{Author Contributions}

Conceptualization, Data acquisition, Formal analysis, Writing - original draft: Minjee Jung. Conceptualization, Formal analysis, Writing - original draft: Seung Yeon Baik. Data acquisition, Writing-original draft: Yourim Kim. Formal analysis, Writing-original draft: Seungkean Kim. Data acquisition: Dogil Min. Formal analysis: Jeong-Youn Kim. Providing experimental program: Seunghee Won. Writing - review \& editing, Supervision: Seung-Hwan Lee.

\section{ORCID}

Minjee Jung https://orcid.org/0000-0002-0938-4798 Seung Yeon Baik https://orcid.org/0000-0002-0218-3889 Yourim Kim https://orcid.org/0000-0002-2809-8877 Sungkean Kim https://orcid.org/0000-0003-4649-6112 Dongil Min Jeong-Youn Kim https://orcid.org/0000-0002-6270-9942

Seunghee Won https://orcid.org/0000-0003-4144-4065

Seung-Hwa Lee https://orcid.org/0000-0002-4858-6173 http://orcid.org/0000-0003-0305-3709

\section{REFERENCES}

1. Aas $\mathrm{M}$, Andreassen $\mathrm{OA}$, Aminoff $\mathrm{SR}$, Færden $\mathrm{A}$, Romm KL, Nesvåg R, et al. A history of childhood trauma is associated with slower improvement rates: findings from a one-year follow-up study of patients with a first-episode psychosis. BMC Psychiatry 2016;16:126.

2. Kohler CG, Turner TH, Bilker WB, Brensinger CM, Siegel SJ,
Kanes SJ, et al. Facial emotion recognition in schizophrenia: intensity effects and error pattern. Am J Psychiatry 2003;160: 1768-1774.

3. Mandal MK, Pandey R, Prasad AB. Facial expressions of emotions and schizophrenia: a review. Schizophr Bull 1998;24: 399-412.

4. Morrison RL, Bellack AS, Mueser KT. Deficits in facial-affect recognition and schizophrenia. Schizophr Bull 1988;14:67-83.

5. Namiki C, Hirao K, Yamada M, Hanakawa T, Fukuyama H, Hayashi T, et al. Impaired facial emotion recognition and reduced amygdalar volume in schizophrenia. Psychiatry Res 2007; 156:23-32.

6. Sachs G, Steger-Wuchse D, Kryspin-Exner I, Gur RC, Katschnig $\mathrm{H}$. Facial recognition deficits and cognition in schizophrenia. Schizophr Res 2004;68:27-35.

7. Wölwer W, Streit M, Polzer U, Gaebel W. Facial affect recognition in the course of schizophrenia. Eur Arch Psychiatry Clin Neurosci 1996;246:165-170.

8. Shenton ME, Dickey CC, Frumin M, McCarley RW. A review of MRI findings in schizophrenia. Schizophr Res 2001;49: 1-52.

9. Jung $\mathrm{W}$, Jang $\mathrm{KI}$, Lee $\mathrm{SH}$. Heart and brain interaction of psychiatric illness: a review focused on heart rate variability, cognitive function, and quantitative electroencephalography. Clin Psychopharmacol Neurosci 2019;17:459-474.

10. Jeon H, Lee SH. From neurons to social beings: short review of the mirror neuron system research and its socio-psychological and psychiatric implications. Clin Psychopharmacol Neurosci 2018;16:18-31.

11. Cho WK, Shin WS, An I, Bang M, Cho DY, Lee SH. Biological aspects of aggression and violence in schizophrenia. 2019; 17:475-486.

12. Goghari VM, Macdonald AW 3rd, Sponheim SR. Temporal lobe structures and facial emotion recognition in schizophrenia patients and nonpsychotic relatives. Schizophr Bull 2011;37:1281-1294.

13. Li H, Chan RC, McAlonan GM, Gong QY. Facial emotion processing in schizophrenia: a meta-analysis of functional neuroimaging data. Schizophr Bull 2010;36:1029-1039.

14. Fried I, MacDonald KA, Wilson CL. Single neuron activity in human hippocampus and amygdala during recognition of faces and objects. Neuron 1997;18:753-765.

15. Adolphs R, Tranel D, Damasio H, Damasio A. Impaired recognition of emotion in facial expressions following bilateral damage to the human amygdala. Nature 1994;372:669-672.

16. Armony JL. Current emotion research in behavioral neuroscience: the role(s) of the amygdala. Emot Rev 2013;5:104-115.

17. Davidson RJ, Irwin W. The functional neuroanatomy of emotion and affective style. Trends Cogn Sci 1999;3:11-21.

18. Haxby JV, Hoffman EA, Gobbini MI. The distributed human neural system for face perception. Trends Cogn Sci 2000;4: 223-233.

19. Furl N, van Rijsbergen NJ, Kiebel SJ, Friston KJ, Treves A, 
Dolan RJ. Modulation of perception and brain activity by predictable trajectories of facial expressions. Cereb Cortex 2010; 20:694-703.

20. Peelen MV, Lucas N, Mayer E, Vuilleumier P. Emotional attention in acquired prosopagnosia. Soc Cogn Affect Neurosci 2009;4:268-277.

21. Trautmann SA, Fehr T, Herrmann M. Emotions in motion: $d y$ namic compared to static facial expressions of disgust and happiness reveal more widespread emotion-specific activations. Brain Res 2009;1284:100-115.

22. Allison T, Puce A, McCarthy G. Social perception from visual cues: role of the STS region. Trends Cogn Sci 2000;4:267-278.

23. Puce A, Allison T, Asgari M, Gore JC, McCarthy G. Differential sensitivity of human visual cortex to faces, letterstrings, and textures: a functional magnetic resonance imaging study. J Neurosci 1996; 16:5205-5215.

24. Steen RG, Mull C, McClure R, Hamer RM, Lieberman JA. Brain volume in first-episode schizophrenia: systematic review and meta-analysis of magnetic resonance imaging studies. Br J Psychiatry 2006;188:510-518.

25. Honea R, Crow TJ, Passingham D, Mackay CE. Regional deficits in brain volume in schizophrenia: a meta-analysis of voxel-based morphometry studies. Am J Psychiatry 2005;162: 2233-2245.

26. Kuroki N, Shenton ME, Salisbury DF, Hirayasu Y, Onitsuka T, Ersner-Hershfield $\mathrm{H}$, et al. Middle and inferior temporal gyrus gray matter volume abnormalities in first-episode schizophrenia: an MRI study. AmJ Psychiatry 2006;163:2103-2110.

27. Lawrie SM, Abukmeil SS. Brain abnormality in schizophrenia. A systematic and quantitative review of volumetric magnetic resonance imaging studies. Br J Psychiatry 1998;172:110120.

28. Nelson MD, Saykin AJ, Flashman LA, Riordan HJ. Hippocampal volume reduction in schizophrenia as assessed by magnetic resonance imaging: a meta-analytic study. Arch Gen Psychiatry 1998;55:433-440.

29. Velakoulis D, Wood SJ, Wong MT, McGorry PD, Yung A, Phillips L, et al. Hippocampal and amygdala volumes according to psychosis stage and diagnosis: a magnetic resonance imaging study of chronic schizophrenia, first-episode psychosis, and ultra-high-risk individuals. Arch Gen Psychiatry 2006;63:139-149.

30. Wright IC, Rabe-Hesketh S, Woodruff PW, David AS, Murray RM, Bullmore ET. Meta-analysis of regional brain volumes in schizophrenia. Am J Psychiatry 2000;157:16-25.

31. Kurtz MM, Richardson CL. Social cognitive training for schizophrenia: a meta-analytic investigation of controlled research. Schizophr Bull 2012;38:1092-1104.

32. Lee KH, Farrow TF, Spence SA, Woodruff PW. Social cognition, brain networks and schizophrenia. Psychol Med 2004; 34:391-400.

33. Arioli M, Crespi C, Canessa N. Social cognition through the lens of cognitive and clinical neuroscience. Biomed Res Int
2018;2018:4283427.

34. Horat SK, Favre G, Prévot A, Ventura J, Herrmann FR, Gothuey I, et al. Impaired social cognition in schizophrenia during the ultimatum game: an EEG study. Schizophr Res 2018;192: 308-316.

35. Sprong M, Schothorst P, Vos E, Hox J, van Engeland H. Theory of mind in schizophrenia: meta-analysis. Br J Psychiatry 2007; 191:5-13.

36. Marwick K, Hall J. Social cognition in schizophrenia: a review of face processing. Br Med Bull 2008;88:43-58.

37. Kosonogov $\mathrm{V}$, Titova A, Vorobyeva E. Empathy, but not mimicry restriction, influences the recognition of change in emotional facial expressions. Q J Exp Psychol (Hove) 2015;68: 2106-2115.

38. Brüne $M$, Abdel-Hamid $M$, Lehmkämper $C$, Sonntag $C$. Mental state attribution, neurocognitive functioning, and psychopathology: what predicts poor social competence in schizophrenia best? Schizophr Res 2007;92:151-159.

39. Fitzpatrick P, Frazier JA, Cochran D, Mitchell T, Coleman C, Schmidt RC. Relationship between theory of mind, emotion recognition, and social synchrony in adolescents with and without autism. Front Psychol 2018;9:1337.

40. Olderbak S, Wilhelm O. Emotion perception and empathy: an individual differences test of relations. Emotion 2017;17: 1092-1106.

41. Lindenmayer JP, McGurk SR, Khan A, Kaushik S, Thanju A, Hoffman L, et al. Improving social cognition in schizophrenia: a pilot intervention combining computerized social cognition training with cognitive remediation. Schizophr Bull 2013;39:507-517.

42. Ashburner J. Computational anatomy with the SPM software. Magn Reson Imaging 2009;27:1163-1174.

43. Ashburner J, Friston KJ. Voxel-based morphometry--the methods. Neuroimage 2000;11:805-821.

44. Ashburner J. A fast diffeomorphic image registration algorithm. Neuroimage 2007;38:95-113.

45. Ashburner J, Friston KJ. Unified segmentation. Neuroimage 2005;26:839-851.

46. Jolliffe D, Farrington DP. Development and validation of the Basic Empathy Scale. J Adolesc 2006;29:589-611.

47. Heider F, Simmel M. An experimental study of apparent behavior. Am J Psychol 1944;57:243-259.

48. Klin A. Attributing social meaning to ambiguous visual stimuli in higher-functioning autism and Asperger syndrome: the social attribution task. J Child Psychol Psychiatry 2000;41:831846.

49. Klin A, Jones W. Attributing social and physical meaning to ambiguous visual displays in individuals with higher-functioning autism spectrum disorders. Brain Cogn 2006;61:40-53.

50. Getz GE, Shear PK, Strakowski SM. Facial affect recognition deficits in bipolar disorder. J Int Neuropsychol Soc 2003;9: 623-632.

51. Park JY, Oh JM, Kim SY, Lee M, Lee C, Kim BR, et al. Korean 
Facial Expressions of Emotion (KOFEE). Seoul: Section of Affect \& Neuroscience, Institute of Behavioral Science in Medicine, Yonsei University College of Medicine;2011.

52. Preacher KJ, Hayes AF. Asymptotic and resampling strategies for assessing and comparing indirect effects in multiple mediator models. Behav Res Methods 2008;40:879-891.

53. Onitsuka T, Shenton ME, Kasai K, Nestor PG, Toner SK, Kikinis $\mathrm{R}$, et al. Fusiform gyrus volume reduction and facial recognition in chronic schizophrenia. Arch Gen Psychiatry 2003; 60:349-355.

54. Feinberg TE, Rifkin A, Schaffer C, Walker E. Facial discrimination and emotional recognition in schizophrenia and affective disorders. Arch Gen Psychiatry 1986;43:276-279.

55. Gur RC, Schroeder L, Turner T, McGrath C, Chan RM, Turetsky $\mathrm{Bl}$, et al. Brain activation during facial emotion processing. Neuroimage 2002;16:651-662.

56. Walker E, McGuire M, Bettes B. Recognition and identification of facial stimuli by schizophrenics and patients with affective disorders. Br J Clin Psychol 1984;23:37-44.

57. Kim DW, Lee $\mathrm{SH}, \mathrm{Im} \mathrm{CH}$. Source activation during facial emotion perception correlates with positive and negative symptoms scores of schizophrenia. Conf Proc IEEE Eng Med Biol Soc 2013;2013:6325-6328.

58. Lee SH, Kim EY, Kim S, Bae SM. Event-related potential patterns and gender effects underlying facial affect processing in schizophrenia patients. Neurosci Res 2010;67:172-180.

59. Aleman A, Kahn RS. Strange feelings: do amygdala abnormalities dysregulate the emotional brain in schizophrenia? Prog Neurobiol 2005;77:283-298.

60. Kosaka H, Omori M, Murata T, lidaka T, Yamada H, Okada T, et al. Differential amygdala response during facial recognition in patients with schizophrenia: an fMRI study. Schizophr Res 2002; 57:87-95.

61. Exner C, Boucsein K, Degner D, Irle E, Weniger G. Impaired emotional learning and reduced amygdala size in schizophrenia: a 3-month follow-up. Schizophr Res 2004;71:493503.

62. Lee JS, Park G, Song MJ, Choi KH, Lee SH. Early visual processing for low spatial frequency fearful face is correlated with cortical volume in patients with schizophrenia. Neuropsychiatr Dis Treat 2015;12:1-14.

63. Maat A, van Haren NEM, Bartholomeusz CF, Kahn RS, Cahn W. Emotion recognition and theory of mind are related to gray matter volume of the prefrontal cortex in schizophrenia. Eur Neuropsychopharmacol 2016;26:255-264.

64. Baas D, Aleman A, Kahn RS. Lateralization of amygdala activation: a systematic review of functional neuroimaging studies. Brain Res Brain Res Rev 2004;45:96-103.

65. Canli T, Zhao Z, Brewer J, Gabrieli JD, Cahill L. Event-related activation in the human amygdala associates with later memory for individual emotional experience. J Neurosci 2000;20: RC99.

66. Hamann S. Cognitive and neural mechanisms of emotional memory. Trends Cogn Sci 2001;5:394-400.

67. Hamann S, Mao H. Positive and negative emotional verbal stimuli elicit activity in the left amygdala. Neuroreport 2002; 13:15-19.

68. Wager TD, Phan KL, Liberzon I, Taylor SF. Valence, gender, and lateralization of functional brain anatomy in emotion: a meta-analysis of findings from neuroimaging. Neuroimage 2003;19:513-531.

69. Aggleton JP, Vann SD, Denby C, Dix S, Mayes AR, Roberts N, et al. Sparing of the familiarity component of recognition memory in a patient with hippocampal pathology. Neuropsychologia 2005;43:1810-1823.

70. Rugg MD, Yonelinas AP. Human recognition memory: a cognitive neuroscience perspective. Trends Cogn Sci 2003;7: 313-319.

71. Yonelinas AP. The nature of recollection and familiarity: a review of 30 years of research. J Mem Lang 2002;46:441-517.

72. Habel U, Koch K, Kellermann T, Reske M, Frommann N, Wölwer W, et al. Training of affect recognition in schizophrenia: Neurobiological correlates. Soc Neurosci 2010;5: 92-104.

73. Luckhaus C, Frommann N, Stroth S, Brinkmeyer J, Wölwer W. Training of affect recognition in schizophrenia patients with violent offences: behavioral treatment effects and electrophysiological correlates. Soc Neurosci 2013;8:505-514.

74. Hooker Cl, Bruce L, Fisher M, Verosky SC, Miyakawa A, D'Esposito M, et al. The influence of combined cognitive plus social-cognitive training on amygdala response during face emotion recognition in schizophrenia. Psychiatry Res 2013; 213:99-107.

75. Horan WP, Kern RS, Green MF, Penn DL. Social cognition training for individuals with schizophrenia: emerging evidence. Am J Psychiatr Rehabil 2008;11:205-252.

76. Popova P, Popov TG, Wienbruch C, Carolus AM, Miller GA, Rockstroh BS. Changing facial affect recognition in schizophrenia: effects of training on brain dynamics. Neuroimage Clin 2014;6:156-165.

77. Silver H, Goodman C, Knoll G, Isakov V. Brief emotion training improves recognition of facial emotions in chronic schizophrenia. A pilot study. Psychiatry Res 2004;128:147154.

78. Wölwer W, Frommann N. Social-cognitive remediation in schizophrenia: generalization of effects of the Training of Affect Recognition (TAR). Schizophr Bull 2011;37 Suppl 2:S63-S70.

79. Wölwer W, Frommann N, Halfmann S, Piaszek A, Streit M, Gaebel W. Remediation of impairments in facial affect recognition in schizophrenia: efficacy and specificity of a new training program. Schizophr Res 2005;80:295-303.

80. Kleinhans NM, Richards T, Weaver K, Johnson LC, Greenson $\mathrm{J}$, Dawson G, et al. Association between amygdala response to emotional faces and social anxiety in autism spectrum disorders. Neuropsychologia 2010;48:3665-3670. 